\title{
On a Boundary-Value Problem for Pseudo-Differential Operators and its Relation to Jump Processes
}

\author{
V. Knopova
}

\begin{abstract}
We show the solvability of Dirichlet and Neumann boundary-value problems in $L_{p}\left(\mathbb{R}_{+}^{n}\right)$ for an operator $A$ of some special type. In case $p=2$ it is possible to give another description of the trace of the domain of $A$ on $\mathbb{R}^{n-1}$.
\end{abstract}

Keywords. Symbol classes, space of generalized smoothness, trace, Dirichlet form, time change, Revuz correspondence

Mathematics Subject Classification (2000). Primary 46E35, 35S15, 60J75, secondary $31 \mathrm{C} 25,47 \mathrm{G} 30$

\section{Introduction}

Consider an operator $A$ with continuous negative definite symbol. Our aim is to find out when $\left(-A, H_{p,+}^{\psi, 2}\right)$ with zero Neumann boundary condition generates an $L_{p}$-sub-Markovian semigroup, and whether it is possible to characterize the process on the boundary. Here $A$ is an operator with continuous negative definite symbol $\psi(\xi), \xi \in \mathbb{R}^{n}$, and $H_{p,+}^{\psi, 2}=\left.H_{p}^{\psi, 2}\left(\mathbb{R}^{n}\right)\right|_{\mathbb{R}_{+}^{n}}$, where $\mathbb{R}_{+}^{n}=\left\{\left(x, x_{n}\right)\right.$ : $\left.x^{\prime} \in \mathbb{R}^{n-1}, x_{n}>0\right\}$, is the restriction to the half-space of the $\psi$-Bessel potential space, see the definitions below. This problem is well known for the diffusion and stable processes, we refer to, for example, [1] and [6]. In this paper we will try to solve the more general problem.

To check whether an operator is the generator of a strongly continuous contraction semigroup we apply the Hille-Yosida theorem. The first problem which we need to overcome is to show the solvability of the boundary value problem

$$
(\lambda+A) u=g, \quad g \in L_{p}\left(\mathbb{R}_{+}^{n}\right),
$$

with some boundary conditions, which in turn depends on the existence of the trace of $H_{p}^{\psi, 2}\left(\mathbb{R}^{n}\right)$ on the boundary $\mathbb{R}^{n-1}$. Therefore first we need show that we

V. Knopova: V. M. Glushkov Institute of Cybernetics, 40 Acad. Glushkov Ave., 03187 Kiev, Ukraine; vic_knopova@yahoo.co.uk 
are allowed to pose such boundary value problem and that the solution to (1.1) exists. For this we need some notions on the spaces of generalized smoothness, associated with the function $\psi$. For such functions of special "locality" type it is possible to show that (1.1) can be posed and it is uniquely solvable.

In case $p=2$ it is known (see [6], or [20]) that if the Dirichlet form associated with operator $-A$ is regular (which is true in our situation) we can associate with $\left(-A, H_{2,+}^{\psi, 2}\right)$ a Markov process. We would like to characterize the associated process on the boundary. It can be characterized as the time changed process with respect to the right inverse of the additive functional, which is in the Revuz correspondence with the measure on the boundary. In [12] it was done for subordinated reflected diffusion for the case when the subordinator is an $\alpha$-stable process. We show that for some generators it is possible to describe the time-changed process in terms of the symbol of the generator of the initial process, and, moreover, to get more simple description of the trace of the domain of this generator on $\mathbb{R}^{n-1}$.

Further we consider a pseudo-differential operator $q(x, D)$ defined for $u \in C_{0}^{\infty}\left(\mathbb{R}^{n}\right)$ :

$$
q(x, D) u(x):=(2 \pi)^{\frac{n}{2}} \int_{\mathbb{R}^{n}} e^{i \xi x} q(x, \xi) \hat{u}(\xi) d \xi,
$$

where $q: \mathbb{R}^{n} \times \mathbb{R}^{n} \rightarrow \mathbb{C}$ is a locally bounded function such that for every $x \in \mathbb{R}^{n}$ the function $q(x, \cdot): \mathbb{R}^{n} \rightarrow \mathbb{C}$ is continuous and negative definite.

Under some conditions on the symbol $q(x, \xi)$ it is possible to specify the domain $D(q(x, D))$ of the operator $q(x, D)$ in $L_{p}\left(\mathbb{R}^{n}\right)$, and $(-q(x, D), D(q(x, D)))$ generates an $L_{p}$-sub-Markovian semigroup. This problem was solved for $p=2$, see $[8,9]$, also [11] and the reference therein. In general case, $1<p<\infty$, the methods which are applicable when $p=2$ do not work, we need stronger estimates on the symbol, i.e., we need to work with symbol classes a bit different from those introduced by W. Hoh, see [8] and [9]. We require a different technique to prove that there exists the unique solution $u \in D(q(x, D))$ to the equation

$$
(\lambda+q(x, D)) u(x)=g(x) \quad \text { for all } g \in L_{p}\left(\mathbb{R}^{n}\right) .
$$

Working with the symbol classes introduced in [2], it is possible to obtain the necessary estimates for $q(x, D)$ and for the symbol $q^{*}(x, \xi)$ of the adjoint operator $q^{*}(x, D)$. Then applying the Fredholm principle we can show that (1.2) is uniquely solvable and the solution belongs to $D(q(x, D))$.

Finally, we show that it is also possible to pose the boundary-value problem (1.1) for some pseudo-differential operators $q(x, D)$. We prove that if $(-q(x, D), D(q(x, D)))$ is a generator of an $L_{p}$-sub-Markovian semigroup on $L_{p}\left(\mathbb{R}^{n}\right)$, then under certain conditions on the symbol $q(x, \xi)$ the operator $\left(-q(x, D),\left.D(q(x, D))\right|_{\mathbb{R}_{+}^{n}}\right)$ generates an $L_{p^{-}}$sub-Markovian semigroup on $L_{p}\left(\mathbb{R}_{+}^{n}\right)$. 


\section{Basic settings}

We start with some notions and results on the spaces of generalized smoothness. The definitions below are taken from [2], see also [3].

Definition 2.1. A sequence $\gamma=\left(\gamma_{j}\right)_{j \in \mathbb{N}_{0}}$ of positive real numbers is called

i) almost increasing if there exists $d_{0}>0$ such that $d_{0} \gamma_{j} \leq \gamma_{k}$ for all $j, k$ such that $0 \leq j \leq k$;

ii) strongly increasing if it is almost increasing and in addition there exists a natural number $\kappa_{0}$ such that

$$
2 \gamma_{j} \leq \gamma_{k} \text { for all } j \text { and } k \text { and } j+\kappa_{0} \leq k \text {; }
$$

iii) of bounded growth if there are positive constants $d_{1}$ and $J_{0} \in \mathbb{N}_{0}$ such that

$$
\gamma_{j+1} \leq d_{1} \gamma_{j} \text { for all } j \geq J_{0}
$$

Let $\left(\sigma_{j}\right)_{j \in \mathbb{N}_{0}}$ be a sequence which satisfies for some $d_{0}, d_{1}>0$ the inequality

$$
d_{0} \sigma_{j} \leq \sigma_{j+1} \leq d_{1} \sigma_{j} \quad \text { for all } j \in \mathbb{N} .
$$

This means that both $\left(\sigma_{j}\right)_{j \in \mathbb{N}_{0}}$ and $\left(\sigma_{j}^{-1}\right)_{j \in \mathbb{N}_{0}}$ are of bounded growth. We will call the sequences which satisfy (2.1) the admissible sequences.

For a fixed strongly increasing sequence $N=\left(N_{j}\right)_{j \in \mathbb{N}_{0}}$ and a fixed $J \in \mathbb{N}$ we define the associated covering $\Omega^{N, J}=\left(\Omega_{j}^{N, J}\right)_{j \in \mathbb{N}_{0}}$ of $\mathbb{R}^{n}$ by

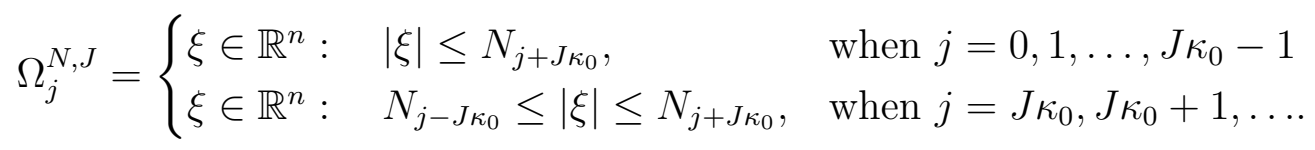

Further, let $\Phi^{N, J}$ be a collection of all function systems $\varphi^{N, J}=\left(\varphi_{j}^{N, J}\right)_{j \in \mathbb{N}_{0}}$ such that

i) $\varphi_{j}^{N, J} \in C_{0}^{\infty}\left(\mathbb{R}^{n}\right), \varphi_{j}^{N, J}(\xi) \geq 0$ for all $\xi \in \mathbb{R}^{n}$, for any $j \in \mathbb{N}_{0}$;

ii) $\operatorname{supp} \varphi_{j}^{N, J} \subset \Omega_{j}^{N, J}$;

iii) for any $\gamma \in \mathbb{N}_{0}^{n}$ there exists $c_{\gamma}>0$ such that for all $j \in \mathbb{N}_{0}$

$$
\left|D^{\gamma} \varphi_{j}^{N, J}(\xi)\right| \leq c_{\gamma}\left(1+|\xi|^{2}\right)^{-\frac{|\gamma|}{2}} \quad \text { for all } \xi \in \mathbb{R}^{n}
$$

iv) there exists a constant $c_{\varphi}>0$ such that

$$
0<\sum_{j=0}^{\infty} \varphi_{j}^{N, J}(\xi)=c_{\varphi}<\infty \quad \text { for all } \xi \in \mathbb{R}^{n}
$$


Definition 2.2. Let $N=\left(N_{j}\right)_{j \in \mathbb{N}_{0}}$ be a strongly increasing sequence, $J \in \mathbb{N}_{0}$, and let $\left(\varphi_{j}^{N, J}\right)_{j \in \mathbb{N}_{j}} \in \Phi^{N, J}$, and $\left(\sigma_{j}\right)_{j \in \mathbb{N}_{0}}$ be an admissible sequence.

i) Let $1<p<\infty, 1 \leq q \leq \infty$. Then the Besov space of generalized smoothness is

$$
B_{p, q}^{\sigma, N}\left(\mathbb{R}^{n}\right)=\left\{u \in S^{\prime}:\|u\|_{B_{p, q}^{\sigma, N}\left(\mathbb{R}^{n}\right)}=\left\|\left(\sigma_{j} \varphi_{j}^{N, J}(D) u\right)_{j \in \mathbb{N}_{0}}\right\|_{l_{q}\left(L_{p}\right)}<\infty\right\}
$$

ii) Let $1<p<\infty, 1<q<\infty$. Then the Triebel-Lizorkin space of generalized smoothness is

$$
F_{p, q}^{\sigma, N}\left(\mathbb{R}^{n}\right)=\left\{u \in S^{\prime}:\|u\|_{F_{p, q}^{\sigma, N}\left(\mathbb{R}^{n}\right)}=\left\|\left(\sigma_{j} \varphi_{j}^{N, J}(D) u\right)_{j \in \mathbb{N}_{0}}\right\|_{L_{p}\left(l_{q}\right)}<\infty\right\}
$$

The spaces (2.2) and (2.3) are the generalizations (due to the standardization theorem) of those introduced by Kaljabin $[14,15]$; they are equivalent (to the spaces from $[14,15])$ if $\sigma$ is strongly increasing and of bounded growth, see [3] for details. As an example, we note, that the sequence $\left(2^{s k}\right)_{k \geq 0}, s>0$, is almost strongly increasing and of bounded growth.

We will need theorems on the existence of the trace space of $F_{p, q}^{\sigma, N}\left(\mathbb{R}^{n}\right)$ and $B_{p, q}^{\sigma, N}\left(\mathbb{R}^{n}\right)$ on some domain $G \subset \mathbb{R}^{n}$ with smooth boundary $\partial G$, and the existence of continuous restriction and extension (i.e., the retraction and the coretraction) to this trace space.

Define (see [15], also [22]) $B_{p q}^{\sigma, N}(G)$ as the space of functions from $B_{p q}^{\sigma, N}\left(\mathbb{R}^{n}\right)$, restricted to $G$, i.e.,

$$
B_{p q}^{\sigma, N}(G)=\left\{u: \text { there exists } g \in B_{p q}^{\sigma, N}\left(\mathbb{R}^{n}\right),\left.g\right|_{G}=u\right\},
$$

with the norm

$$
\|u\|_{B_{p q}^{\sigma, N}(G)}=\inf _{g \in B_{p q}^{\sigma, N}\left(\mathbb{R}^{n}\right),\left.g\right|_{G}=u}\|g\|_{B_{p q}^{\sigma, N}\left(\mathbb{R}^{n}\right)} .
$$

Analogously one can define the space $F_{p q}^{\sigma, N}(G)$.

Suppose that $\left(\beta_{j}\right)_{j \in \mathbb{N}_{0}}$ is a strongly increasing sequence of bounded growth, and let $1 \leq m<n$ be an integer. Denote

$$
\alpha_{j}=\beta_{j} N_{j}^{\frac{m-n}{p}} .
$$

Theorem 2.3 ([16, Theorem 5.1]). The condition $\left(\alpha_{j}^{-1}\right)_{j \in \mathbb{N}_{0}} \in l_{p^{\prime}}$ is necessary and sufficient for the existence of retraction from $F_{p, q}^{\beta, N}\left(\mathbb{R}^{n}\right)$ to $B_{p, p}^{\alpha, N}\left(\mathbb{R}^{m}\right)$ with the corresponding coretraction.

For the next statement we refer to [15, Theorem 1 and Lemma 1] and [16, Lemma 7.2]. 
Theorem 2.4. Let $G \subset \mathbb{R}^{n}, \partial G$ be smooth. There exists the coretraction from $F_{p, q}^{\beta, N}(G)$ to $F_{p, q}^{\beta, N}\left(\mathbb{R}^{n}\right)$.

Note that the restriction can be defined in the natural way: $\operatorname{Re} u(x)=\left.u\right|_{G}$, and it is continuous:

$$
\|\operatorname{Re} u\|_{F_{p, q}^{\beta, N}(G)} \leq c\|u\|_{F_{p, q}^{\beta, N}\left(\mathbb{R}^{n}\right)},
$$

which follows from the definition of the norm in $F_{p, q}^{\beta, N}(G)$.

Definition 2.5. Let $\mathcal{A}$ be the class of all non-negative $C^{\infty}$-functions $a: \mathbb{R}^{n} \rightarrow \mathbb{R}$ with the following properties:

A. $\lim _{|\xi| \rightarrow \infty} a(\xi)=\infty$;

B. $a(\xi)$ is almost increasing in $|\xi|$, i.e., there exist constants $\delta_{0} \geq 1$ and $R>0$ such that $a(\xi) \leq \delta_{0} a(\eta)$ if $R \leq|\xi| \leq|\eta|$;

C. there exists $m>0$ such that $a(\xi)|\xi|^{-m}$ is almost decreasing in $|\xi|$, i.e., there exists a constant $\delta_{m}, 0<\delta_{m} \leq 1$, and $R>0$ such that

$$
a(\xi)|\xi|^{-m} \geq \delta_{m} a(\eta)|\eta|^{-m}, \quad \text { if } R \leq|\xi| \leq|\eta|
$$

D. for every multi-index $\alpha=\left(\alpha_{1}, \ldots, \alpha_{n}\right), \alpha_{i} \in \mathbb{N} \cup\{0\}, i=1, \ldots, n$, there exists some $c_{\alpha}>0$ such that

$$
\left|D^{\alpha} a(\xi)\right| \leq c_{\alpha} a(\xi)\left(1+|\xi|^{2}\right)^{-\frac{|\alpha|}{2}}, \quad \text { if }|\xi| \geq R .
$$

The functions from $\mathcal{A}$ are called admissible functions.

Later on we will need condition

E. there exists $r \in[0,1)$ such that the function $\xi \rightarrow a(\xi)\left(1+|\xi|^{2}\right)^{-\frac{r}{2}}$ is increasing in $|\xi|$.

It was proved in [3] that for an admissible function $a(\xi)$ the sequence $N^{a}=$ $\left(N_{j}^{a}\right)_{j \in \mathbb{N}_{0}}$, where

$$
N_{j}^{a}=\sup \left\{|\xi|: a(\xi) \leq 2^{2 j}\right\}, \quad j \in \mathbb{N}_{0},
$$

is strongly increasing, see Lemma 3.1.17 from [3]. Note that the definition of the strongly increasing sequence by (2.5) does not require the radial symmetry of the symbol.

Let $\psi$ be a real-valued continuous negative definite function with representation

$$
\psi(\xi)=\int_{\mathbb{R}^{n} \backslash\{0\}}(1-\cos (\xi \cdot y)) \nu(d y),
$$


where $\nu(d y)$ is a Lévy measure which satisfies $\int_{\mathbb{R}^{n} \backslash\{0\}}\left(1 \wedge|y|^{2}\right) \nu(d y)<\infty$, and consider the generalized $\psi$-Bessel potential space

$$
H_{p}^{\psi, s}\left(\mathbb{R}^{n}\right)=\left\{u \in S^{\prime}\left(\mathbb{R}^{n}\right):\left\|F^{-1}\left((1+\psi(\xi))^{\frac{s}{2}} \hat{u}(\xi)\right)\right\|_{p}<\infty\right\},
$$

where $s>0,1<p<\infty$. We refer to [4] or [11] for more details about such spaces.

Suppose in addition that $\psi$ is an admissible function, and let $N^{\psi}=\left(N_{j}^{\psi}\right)_{j \geq 0}$ be the strongly increasing sequence associated with $\psi$. Then the spaces $H_{p}^{\psi, s}\left(\mathbb{R}^{n}\right)$ and $F_{p, 2}^{\sigma^{s}, N^{\psi}}\left(\mathbb{R}^{n}\right)$ coincide, $\sigma^{s}=\left(2^{j s}\right)_{j \in \mathbb{N}_{0}}$, see [3] for details. We use the notation $H_{p}^{\psi, s}$ for $H_{p}^{\psi, s}\left(\mathbb{R}^{n}\right)$ when it is clear which space is meant.

Let $A$ be an operator with a continuous negative definite symbol $\psi$. On $C_{0}^{\infty}\left(\mathbb{R}^{n}\right), A$ admits the representation $([10])$

$$
A u(x)=\int_{\mathbb{R}^{n} \backslash\{0\}}\left(u(x-y)-u(x)-\sum_{j=1}^{n} \frac{y_{j}}{1+|y|^{2}} \frac{\partial u(x)}{\partial x_{j}}\right) \nu(d y) .
$$

It was proved (see [4]) that for real-valued $\psi$ the domain of $A$ in $L_{p}$ is $H_{p}^{\psi, 2}$, the operator $A$ is an isomorphism between $H_{p}^{\psi, s-2}$ and $H_{p}^{\psi, s},-\infty<s<\infty$, and $\left(-A, H_{p}^{\psi, 2}\right)$ is a generator of an $L_{p}$-sub-Markovian semigroup.

Let

with the norm

$$
H_{p,+}^{\psi, s}=\left\{u: \text { there exists } g \in H_{p}^{\psi, s},\left.g\right|_{\mathbb{R}_{+}^{n}}=u\right\} .
$$

$$
\|g\|_{H_{p,+}^{\psi, s}}=\inf \|f\|_{H_{p}^{\psi, s}}
$$

where the infimum is taken over all such extensions (note that $H_{p,+}^{\psi, 2}$ coincides with $\left.F_{p, 2}^{\sigma^{2}, N^{\psi}}\left(\mathbb{R}_{+}^{n}\right), \sigma^{2}=\left(2^{2 j}\right)_{j \geq 0}\right)$. In this case the operator, defined as $\operatorname{Re} u(x)=\left\{u\left(x^{\prime}, 0\right), \frac{\partial u}{\partial x_{n}}\left(x^{\prime}, 0\right)\right\}$ is the retraction from $H_{p}^{\psi, 2}\left(\mathbb{R}^{n}\right)=F_{p, 2}^{\sigma^{2}, N^{\psi}}\left(\mathbb{R}^{n}\right)$ to $B_{p, p}^{\alpha^{(1)}, N^{\psi}}\left(\mathbb{R}^{n-1}\right) \times B_{p, p}^{\alpha^{(2)}, N^{\psi}}\left(\mathbb{R}^{n-1}\right)$, where $\alpha_{j}^{(1)}=2^{2 j}\left(N_{j}^{\psi}\right)^{-\frac{1}{p}}, \alpha_{j}^{(2)}=2^{2 j}\left(N_{j}^{\psi}\right)^{-1-\frac{1}{p}}$, and there exists the corresponding coretraction.

Let $\mathbb{R}_{0+}^{n}=\left\{\left(x^{\prime}, x_{n}\right): x^{\prime} \in \mathbb{R}^{n-1}, x_{n} \geq 0\right\}$. Later on we will also need the space

$$
\tilde{H}_{p,+}^{\psi, s}=\left\{u: u \in H_{p}^{\psi, s}, \operatorname{supp} u \subset \mathbb{R}_{0+}^{n}\right\} .
$$

Here and below we understand $\operatorname{supp} u \subset K, K$ is a closed set, in the sense of distributions, i.e., $\int_{\mathbb{R}^{n}} u(x) \varphi(x) d x=0$ for any $\varphi \in S\left(\mathbb{R}^{n}\right)$, $\operatorname{supp} \varphi \subset \mathbb{R}^{n} \backslash K$.

Consider some examples.

Example 2.6. Let $\psi(\xi)=\ln (1+|\xi|)$. Then

$$
N_{j}^{\psi}=\sup \left\{|\xi|: \ln (1+|\xi|) \leq 2^{2 j}\right\}=\sup \left\{|\xi|:|\xi| \leq e^{2^{2 j}}-1\right\}=e^{2^{2 j}}-1,
$$

and thus $\alpha_{j}=\frac{2^{2 j}}{\left(e^{2^{2 j}}-1\right)^{\frac{1}{p}}}$. Clearly, $\left(\alpha_{j}^{-1}\right)_{j \in \mathbb{N}_{0}}$ does not converge in $l_{p}$ for any $p$, $1 \leq p<\infty$. 
Example 2.7. Let $\psi(\xi)=|\xi|^{\beta} \ln (1+|\xi|)$, and $0<\beta<1$. Then

$$
N_{j}^{\psi}=\sup g\left\{|\xi|:|\xi|^{\beta} \ln (1+|\xi|) \leq 2^{2 j}\right\} \leq \sup \left\{|\xi|:|\xi|^{\beta} \leq 2^{2 j}\right\}=2^{2 \frac{j}{\beta}} \text {. }
$$

Then $\alpha_{j}^{-1} \leq 2^{-2 j\left(1-\frac{1}{p \beta}\right)}$, and the sequence $\left(\alpha_{j}^{-1}\right)_{j \in \mathbb{N}_{0}}$ is convergent in $l_{p}$ for $\beta>\frac{1}{p}$.

We will also need some symbol classes, as well as some results from symbolic calculus. The following definition and the theorem are taken from $[2$, Chapter 16].

Definition 2.8. Let $\psi: \mathbb{R}^{n} \rightarrow \mathbb{R}$ be an admissible continuous negative definite function which satisfies E. Let $\mu \in \mathbb{R}$ and $0 \leq \delta \leq 1$. Then let $S_{1, \delta}^{\psi, \mu}$ be the collection of all complex valued $C^{\infty}$ functions $a: \mathbb{R}^{n} \times \mathbb{R}^{n} \rightarrow \mathbb{C}$ such that for any multi-indices $\beta$ and $\alpha$ there exists a constant $c_{\alpha \beta}>0$ such that

$$
\left|D_{x}^{\beta} D_{\xi}^{\alpha} a(x, \xi)\right| \leq c_{\alpha \beta}(1+\psi(\xi))^{\frac{\mu}{2}}\left(1+|\xi|^{2}\right)^{\frac{-|\alpha|+\delta|\beta|}{2}} \quad \text { for any } x, \xi \in \mathbb{R}^{n} .
$$

Theorem 2.9 ([2, Theorems 16.3.5/16.3.7]). Let $\psi: \mathbb{R}^{n} \rightarrow \mathbb{R}$ be an admissible continuous negative definite function which satisfies $\mathrm{E}, \mu \in \mathbb{R}, 0 \leq \delta<1$, and $a \in S_{1, \delta}^{\psi, \mu}$. For $1<p<\infty$ and $s \in \mathbb{R}$ the pseudo-differential operator $a(\cdot, D)$ is continuous from the space $H_{p}^{\psi, s+\mu}$ into $H_{p}^{\psi, s}$, i.e., for any $u \in H_{p}^{\psi, s+\mu}$ it holds

$$
\|a(x, D) u\|_{H_{p}^{\psi, s}} \leq c\|u\|_{H_{p}^{\psi, s+\mu}} .
$$

For simplicity we will consider only the symbols from $S_{1,0}^{\psi, \mu}, \mu \in \mathbb{R}$, i.e., with $\delta=0$. Although we will not work with the spaces $S_{\rho}^{m, \psi}$, for our convenience we give the definition of these spaces.

Definition 2.10. Let $m \in \mathbb{R}$ and $\psi$ be a continuous negative definite function such that for all $\alpha \in \mathbb{N}_{0}^{n}$ it holds

$$
\left|\partial_{\xi}^{\alpha}(1+\psi(\xi))^{\frac{m}{2}}\right| \leq c_{|\alpha|}(1+\psi(\xi))^{\frac{m-\rho(|\alpha|)}{2}},
$$

where $\rho: \mathbb{N}_{0} \rightarrow \mathbb{N}_{0}, \rho(k):=\min (k, 2)$. We call a $C^{\infty}$-function $q: \mathbb{R}^{n} \times \mathbb{R}^{n} \rightarrow \mathbb{C}$ a symbol in the class $S_{\rho}^{m, \psi}$ if for all $\alpha, \beta \in \mathbb{N}_{0}^{n}$ there are constants $c_{\alpha \beta} \geq 0$ such that

$$
\left|\partial_{\xi}^{\alpha} \partial_{x}^{\beta} q(x, \xi)\right| \leq c_{\alpha \beta}(1+\psi(\xi))^{\frac{m-\rho(|\alpha|)}{2}} .
$$

Clearly, for admissible $\psi$ we have $S_{1,0}^{\psi, m} \subset S_{\rho}^{m, \psi}$. We refer for the definitions and some results below to [11, Chapter 2.4], and also to the original papers $[8,9]$.

Definition 2.11. The class $\Sigma$ consists of all complex valued $C^{\infty}$-functions $a: \mathbb{R}^{n} \times \mathbb{R}^{n} \rightarrow \mathbb{C}$ such that for all $\alpha, \beta \in \mathbb{N}_{0}^{n}$ and all $\eta, y \in \mathbb{R}^{n}$ the estimate

$$
\left|\partial_{\eta}^{\alpha} \partial_{y}^{\beta} a(\eta, y)\right| \leq c_{\alpha \beta}\left(1+|\eta|^{2}\right)^{\frac{m+\delta|\beta|}{2}}\left(1+|y|^{2}\right)^{\frac{\tau}{2}}
$$


holds for some $m \in \mathbb{R}, \delta \in[0,1)$ and $\tau \geq 0$. For all $a \in \Sigma$ and $\chi \in S\left(\mathbb{R}^{n} \times \mathbb{R}^{n}\right)$ such that $\chi(0,0)=1$ we define the oscillatory integral by

$$
O_{s}-\int_{\mathbb{R}^{n}} \int_{\mathbb{R}^{n}} e^{-i y \cdot \eta} a(\eta, y) d y d \eta:=\lim _{\varepsilon \rightarrow 0} \int_{\mathbb{R}^{n}} \int_{\mathbb{R}^{n}} e^{-i y \cdot \eta} \chi(\varepsilon \eta, \varepsilon \eta) a(\eta, y) d y d \eta
$$

Theorem 2.12. For all $a \in \Sigma$ the oscillatory integral (2.11) exists, it is independent of the choice of $\chi$ and

$$
\begin{aligned}
& O_{s}-\int_{\mathbb{R}^{n}} \int_{\mathbb{R}^{n}} e^{-i y \cdot \eta} a(\eta, y) d y d \eta \\
& =\int_{\mathbb{R}^{n}} \int_{\mathbb{R}^{n}} e^{-i y \cdot \eta}\left(1+|y|^{2}\right)^{-l^{\prime}}\left(1-\triangle_{\eta}\right)^{l^{\prime}}\left\{\left(1+|\eta|^{2}\right)^{-l}\left(1-\triangle_{y}\right)^{l} a(\eta, y)\right\} d y d \eta
\end{aligned}
$$

holds for $l, l^{\prime} \in \mathbb{N}$, such that $-2 l(1-\delta)+m<-n,-2 l^{\prime}+\tau<-n$, where $\delta, \tau$ and $m$ are from equation (2.10).

In the same fashion as the classes $S_{0}^{m, m^{\prime}, \psi}$ were introduced in [8], we introduce the classes $S_{1,0}^{\psi, m, m^{\prime}}$.

Definition 2.13. Let $\psi$ be an admissible continuous negative definite function, $m, m^{\prime} \in \mathbb{R}$. The class $S_{1,0}^{\psi, m, m^{\prime}}$ of double symbols of orders $m$ and $m^{\prime}$ consists of all functions $q\left(x, \xi ; x^{\prime}, \xi^{\prime}\right): \mathbb{R}^{n} \times \mathbb{R}^{n} \times \mathbb{R}^{n} \times \mathbb{R}^{n} \rightarrow \mathbb{C}, n$ times differentiable in $\xi$ and $\xi^{\prime}$, and $r$ times in $x$ and $x^{\prime}, r \in \mathbb{N}$, such that

$$
\left|\partial_{\xi}^{\alpha} \partial_{x}^{\beta} \partial_{\xi^{\prime}}^{\alpha^{\prime}} \partial_{x^{\prime}}^{\beta^{\prime}} q\left(x, \xi ; x^{\prime}, \xi^{\prime}\right)\right| \leq c_{\alpha \beta \alpha^{\prime} \beta^{\prime}} \frac{(1+\psi(\xi))^{\frac{m}{2}}}{\left(1+|\xi|^{2}\right)^{\frac{|\alpha|}{2}}} \frac{\left(1+\psi\left(\xi^{\prime}\right)\right)^{\frac{m^{\prime}}{2}}}{\left(1+\left|\xi^{\prime}\right|^{2}\right)^{\frac{\left|\alpha^{\prime}\right|}{2}}}
$$

for all $0 \leq|\alpha|,\left|\alpha^{\prime}\right| \leq n, 0 \leq|\beta|,\left|\beta^{\prime}\right| \leq r$.

In the end of this section we formulate the Hille-Yosida theorem which gives us the necessary and sufficient conditions when a closed operator generates a strongly contraction semigroup. Denote by $R(A)$ the range of an operator $A$.

Theorem 2.14 (Hille-Yosida theorem). A closed linear operator $(A, D(A))$ on a Banach space $\left(X,\|\cdot\|_{X}\right)$ is the generator of a strongly continuous contraction semigroup $\left(T_{t}\right)_{t \geq 0}$ if and only if the following conditions hold:

1. $D(A) \subset X$ is dense;

2. A is a dissipative operator;

3. $R(\lambda-A)=X$ for some $\lambda>0$.

Let $X=L_{p}\left(\mathbb{R}^{n}\right)$. A strongly continuous contraction semigroup $\left(T_{t}\right)_{t \geq 0}$ is sub-Markovian, if its generator $A$ is a Dirichlet operator: for $u \in D(A)$

$$
\int_{\mathbb{R}^{n}} A u(x)\left((u(x)-1)^{+}\right)^{p-1} d x \leq 0 .
$$

For pseudo-differential operators with negative definite symbols this condition is satisfied, see $\S 4.6$ from [10], also $\S 2.6$ from [11]. 


\section{The solvability of the boundary-value problem}

In this Chapter we prove the solvability of boundary value problems in $\mathbb{R}_{+}^{n}$ for some operator with continuous negative definite symbol, and we show that this operator is a generator of an $L_{p^{-}}$sub-Markovian semigroup in $L_{p}\left(\mathbb{R}_{+}^{n}\right)$.

Consider an operator $A$ with symbol $\psi_{0}(\xi), \xi=\left(\xi^{\prime}, \xi_{n}\right), \xi^{\prime} \in \mathbb{R}^{n-1}, \xi_{n} \in \mathbb{R}$, which satisfies the following condition:

F. For $z \in \mathbb{C}, \operatorname{Im} z>0$ there exists an extension $\psi_{0}\left(\xi^{\prime}, z\right)$ of the function $\psi_{0}\left(\xi^{\prime}, \xi_{n}\right)$, which is analytic in $z$, and $\left|\lambda+\psi_{0}\left(\xi^{\prime}, z\right)\right|>0$ for some $\lambda>0$.

Under such a condition we obtain by the same method, used in Theorem 2.10.3/1 from [22], that $\lambda+A$ is an isomorphism between $H_{p,+}^{\psi, 2}$ and $L_{p}\left(\mathbb{R}_{+}^{n}\right)$. We postpone the proof of this fact to Section 6 , where we give the proof for a wider class of pseudo-differential operators.

In addition, suppose that $\psi_{0} \in S_{1,0}^{\psi, 2}$ for some admissible continuous negative definite function $\psi$, which satisfies E. Under this condition $D(A)=H_{p}^{\psi, 2}$ is closed with respect to the graph norm of $A$. Sometimes we can easily determine $\psi$. For example, let $\psi_{0}(\xi)=\left(\phi\left(\xi^{\prime}\right)+i \xi_{n}\right)^{\alpha}, \xi=\left(\xi^{\prime}, \xi_{n}\right) \in \mathbb{R}^{n}, 0<\alpha<1$, and $\phi$ be an admissible real-valued continuous negative definite function. It can be shown that $\psi(\xi)=\operatorname{Re} \psi_{0}(\xi)$, see [18] or [17]. For $p=2$ we even don't need the condition $\psi_{0} \in S_{1,0}^{\psi, 2}\left(\right.$ for $\left.\psi(\xi)=\operatorname{Re} \psi_{0}(\xi)\right)$, since we always have $D(A)=H^{\psi, 2}$, if $\psi_{0}$ satisfies the sector condition

$$
\left|\operatorname{Im} \psi_{0}\right| \leq c \operatorname{Re} \psi_{0}
$$

We will come back to this problem later in Section 5, for an operator with more general symbol $q(x, \xi)$.

Consider the boundary value problem

$$
(\lambda+A) u(x)=g(x), \quad x \in \mathbb{R}_{+}^{n},
$$

with Dirichlet

$$
u\left(x^{\prime}, 0\right)=h\left(x^{\prime}\right), \quad x^{\prime} \in \mathbb{R}^{n-1},
$$

or Neumann

$$
\frac{\partial u}{\partial x_{n}}\left(x^{\prime}, 0\right)=r\left(x^{\prime}\right), \quad x^{\prime} \in \mathbb{R}^{n-1}
$$

boundary conditions. Denote further by $\|\cdot\|_{p,+}$ the norm in $L_{p}\left(\mathbb{R}_{+}^{n}\right)$.

Theorem 3.1. Let $\psi_{0}$ be a continuous negative definite function, satisfying condition $\mathrm{F}$, and such that $\psi_{0} \in S_{1,0}^{\psi, 2}$ for some admissible continuous negative definite function $\psi$, which satisfies $\mathrm{E}$. Let $\left(-A, H_{p,+}^{\psi, 2}\right)$ be an operator with symbol $\psi_{0}, \alpha_{j}^{(1)}=2^{2 j}\left(N_{j}^{\psi}\right)^{-\frac{1}{p}}, \alpha_{j}^{(2)}=2^{2 j}\left(N_{j}^{\psi}\right)^{-1-\frac{1}{p}}$, where $N_{j}^{\psi}$ is defined in (2.5). 
a) If $\left(\left(\alpha_{j}^{(1)}\right)^{-1}\right)_{j \in \mathbb{N}_{0}} \in l_{p^{\prime}}$, then the boundary value problem (3.2), (3.3) is uniquely solvable for any $h \in B_{p p}^{\alpha^{(1)}, N^{\psi}}\left(\mathbb{R}^{n-1}\right)$.

b) If $\left(\left(\alpha_{j}^{(2)}\right)^{-1}\right)_{j \in \mathbb{N}_{0}} \in l_{p^{\prime}}$, then the boundary value problem (3.2), (3.4) is uniquely solvable for any $r \in B_{p p}^{\alpha^{(2)}, N^{\psi}}\left(\mathbb{R}^{n-1}\right)$.

Proof. From the structure of the symbol $\psi_{0}$ we see, that we are allowed to pose the Dirichlet or Neumann boundary conditions.

We prove that the boundary value problem (3.2) with the Dirichlet or Neumann boundary conditions is uniquely solvable. Since $A$ is an isomorphism from $H_{p,+}^{\psi, 2}$ to $L_{p}\left(\mathbb{R}_{+}^{n}\right)$ (see Section 6 for the proof in the more general situation), then the equation $(\lambda+A) u_{0}=g$ is uniquely solvable in the class $\tilde{H}_{p,+}^{\psi, 2}$ for all $g \in L_{p}\left(\mathbb{R}_{+}^{n}\right)$. The space $\tilde{H}_{p,+}^{\psi, 2}$ is a closed subspace of $H_{p}^{\psi, 2}$, therefore $H_{p}^{\psi, 2}=\tilde{H}_{p,+}^{\psi, 2} \oplus H_{p,-}^{\psi, 2}$, i.e., each $u \in H_{p}^{\psi, 2}$ can be uniquely decomposed as $u=u_{0}+u_{1}$, where $u_{0} \in \tilde{H}_{p,+}^{\psi, 2}$, and $u_{1} \in H_{p,-}^{\psi, 2}$.

Consider the Dirichlet boundary value problem (3.2), (3.3) with $u\left(x^{\prime}, 0\right)=$ $h\left(x^{\prime}\right)$ (we can proceed similarly in the case of Neumann boundary condition). Let $v$ be a continuous extension of $h$ to $H_{p,-}^{\psi, 2}, u=u_{0}+v$, and denote by $\tilde{u}$ the function, on which the infimum $\inf _{v}\left\{\|u\|_{H_{p}^{\psi, 2}}:\|u\|_{H_{p}^{\psi, 2}}<\infty\right\}$ is attained, and $\tilde{v}=\arg \inf _{v}\left\{\|u\|_{H_{p}^{\psi, 2}}:\|u\|_{H_{p}^{\psi, 2}}<\infty\right\}$. Then $\tilde{u}=u_{0}+\tilde{v}$. We will show below that $\|\tilde{u}\|_{H_{p,+}^{\psi, 2}}<\infty$. Since $\tilde{v}\left(x^{\prime}, 0\right)=h\left(x^{\prime}\right)$, then $\tilde{u}\left(x^{\prime}, 0\right)=h\left(x^{\prime}\right)$. We have

$$
(\lambda+A) \tilde{u}=(\lambda+A) u_{0}=g, \quad x \in \mathbb{R}_{+}^{n} .
$$

Therefore the boundary value problem (3.2), (3.3) (or (3.2), (3.4)) is uniquely solvable and it remains to show that the solution belongs to $H_{p,+}^{\psi, 2}$.

On $\mathbb{R}^{n}$ we have the inequality (see [4], also [11])

$$
c_{1}\|u\|_{H_{p}^{\psi, 2}} \leq\|A u\|_{L_{p}}+\|u\|_{L_{p}} \leq c_{2}\|u\|_{H_{p}^{\psi, 2}} .
$$

Let $w \in H_{p,+}^{\psi, 2}$. Then $w(\cdot, 0) \in B_{p p}^{\alpha^{(1)}, N^{\psi}}\left(\mathbb{R}^{n-1}\right)\left(\right.$ or $\left.\frac{\partial w(\cdot, 0)}{\partial x_{n}} \in B_{p p}^{\alpha^{(2)}, N^{\psi}}\left(\mathbb{R}^{n-1}\right)\right)$, and suppose that

We also have

$$
\frac{\partial^{j} \tilde{u}\left(x^{\prime}, 0\right)}{\partial x_{n}^{j}}=\frac{\partial^{j} w\left(x^{\prime}, 0\right)}{\partial x_{n}^{j}}, \quad j=0,1
$$

$$
\|w\|_{H_{p,+}^{\psi, 2}} \leq c_{1}\|w(\cdot, 0)\|_{B_{p p}^{\alpha(1), N \psi}}, \quad\|w\|_{H_{p,+}^{\psi, 2}} \leq c_{2}\left\|\frac{\partial w(\cdot, 0)}{\partial x_{n}}\right\|_{B_{p p}^{\alpha(2), N \psi}} .
$$

Since $\operatorname{supp}(\tilde{u}-w) \subset \mathbb{R}_{0+}^{n}$, we obtain, because the inequalities (3.5) hold for functions with support in $\mathbb{R}_{0+}^{n}$,

$$
\begin{aligned}
\|\tilde{u}\|_{H_{p,+}^{\psi, 2}} & \leq\|\tilde{u}-w\|_{H_{p,+}^{\psi, 2}}+\|w\|_{H_{p,+}^{\psi, 2}} \\
& \leq c_{3}\left(\|A(\tilde{u}-w)\|_{p,+}+\|\tilde{u}-w\|_{p,+}+\|w\|_{H_{p,+}^{\psi, 2}}\right) \\
& \leq c_{4}\left(\|A \tilde{u}\|_{p,+}+\|\tilde{u}\|_{p,+}+\|w\|_{H_{p,+}^{\psi, 2}}\right),
\end{aligned}
$$


where in the last line we used that by the definition of the norm in $H_{p,+}^{\psi, s}$

$$
\|A w\|_{p,+}+\|w\|_{p,+} \leq c\|w\|_{H_{p,+}^{\psi, 2}}
$$

Finally, using (3.6), we arrive at

$$
c_{5}\|\tilde{u}\|_{H_{p,+}^{\psi, 2}} \leq\left(\|A \tilde{u}\|_{p,+}+\|\tilde{u}\|_{p,+}+\left\|\frac{\partial^{j} \tilde{u}(\cdot, 0)}{\partial x_{n}^{j}}\right\|_{B_{p p}^{\alpha(j), N^{\psi}}}\right) \leq c_{6}\|\tilde{u}\|_{H_{p,+}^{\psi, 2}}
$$

for $j=0,1$ (the right-hand side follows from the existence of continuous restriction). But (3.7) shows that the solution to (3.2) with boundary conditions (3.3) or (3.4) belongs to $H_{p,+}^{\psi, 2}$.

Theorem 3.2. Let $A$ be an operator with continuous negative definite symbol, which satisfies the conditions of Theorem 3.1. Then the operator $\left(-A, H_{p,+}^{\psi, 2}\right)$ is the generator of an $L_{p}$-sub-Markovian semigroup.

Proof. We need to check the conditions of Hille-Yosida theorem.

1. The space $H_{p,+}^{\psi, 2}$ is dense in $L_{p}\left(\mathbb{R}_{+}^{n}\right)$.

2. Since $-A$ is a Dirichlet operator on $H_{p}^{\psi, 2}$ (see [11], for example), then by the definition of $H_{p,+}^{\psi, 2}$ it is a Dirichlet operator on $H_{p,+}^{\psi, 2}$.

3. The solvability of (3.2) follows from Theorem 3.1.

\section{Dirichlet forms and the boundary process}

Suppose we can construct a Markov process moving in $\mathbb{R}_{+}^{n}$. Our next aim is to describe the jump process on the boundary. To do this we consider the $L_{2}$-setting, and use the methods from the theory of symmetric Dirichlet forms. We start with some general notions, see [6] and [5] for details.

Let $(X, \mathcal{B}, m)$ be a locally compact measure space, and $m$ be a positive Radon measure such that $\operatorname{supp} m=X$. Consider the Hilbert space $L_{2}(X, m)$. A form $\mathcal{E}$ is a symmetric form on $L_{2}(X, m)$ if

a) $\mathcal{E}: \mathcal{F} \times \mathcal{F} \rightarrow \mathbb{R}$, and $\mathcal{F}$ is dense in $L_{2}\left(\mathbb{R}^{n}\right)$;

b) $\mathcal{E}(u, v)=\mathcal{E}(v, u), \mathcal{E}(u+v, w)=\mathcal{E}(u, w)+\mathcal{E}(v, w), a \mathcal{E}(u, v)=\mathcal{E}(a u, v)$, $\mathcal{E}(u, u) \geq 0$ for $u, v, w \in \mathcal{F}$ and $a \in \mathbb{R}$.

The space $\mathcal{F}$ is called the domain of $\mathcal{E}$.

The Dirichlet form is a symmetric, closed and Markovian form on $L_{2}(X, m)$, where Markovian mean that

$$
\text { if } u \in \mathcal{F}, v=(0 \vee u) \wedge 1, \quad \text { then } v \in \mathcal{F}, \mathcal{E}(v, v) \leq \mathcal{E}(u, u) .
$$

The Dirichlet form $(\mathcal{E}, \mathcal{F})$ is called regular, if there exists a subset $C$ which is dense in $\mathcal{F} \cap C_{0}(X)$, where $C_{0}(X)$ is a space of continuous on $X$ functions 
with compact support. It is known (see, for example, [6, Chapter 7]), that regular Dirichlet forms and Hunt processes (i.e., strong Markov processes whose sample paths are right continuous and quasi-left continuous) are in one-to-one correspondence, and therefore studying a regular Dirichlet form we can get the information about the behaviour of the corresponding Hunt process. One can construct the symmetric Dirichlet form starting with a self-adjoint Dirichlet operator, i.e., if $-B$ is a self-adjoint Dirichlet operator, then

$$
\mathcal{E}_{\lambda}(u, v)=(\sqrt{-B} u, \sqrt{-B} v)+\lambda(u, v)
$$

is the corresponding Dirichlet form, and $\mathcal{F}=D(\sqrt{-B})$ (here $(\cdot, \cdot)$ is a scalar product in $\left.L_{2}(X, m)\right)$. We will use the notation $\mathcal{E}(\cdot, \cdot)$ for $\mathcal{E}_{0}(\cdot, \cdot)$.

Denote by $\mathcal{F}_{e}$ the family of $m$-measurable functions $u$ on $X$ such that $|u|<\infty$ m-a.e., and there exists an $\mathcal{E}$-Cauchy sequence $\left\{u_{n}\right\}_{n \geq 0} \in \mathcal{F}$ such that $\lim _{n \rightarrow \infty} u_{n}=u$ m-a.e.. $\left(\mathcal{E}, \mathcal{F}_{e}\right)$ is called the extended Dirichlet space of $(\mathcal{E}, \mathcal{F})$, and under such a completion it is a Hilbert space (note that $\left(\mathcal{E}_{\lambda}, \mathcal{F}\right)$ is complete, so $\mathcal{F}=\mathcal{F}_{e}$ ).

We need the notions on the capacity of a set. Let $\mathcal{O}$ be a family of open sets in $L_{2}(X, m)$, and $\mathcal{L}_{C}=\{u \in \mathcal{F}, u \geq 1$ a.e. on $C\}$. The set function

$$
\operatorname{Cap}(C)= \begin{cases}\inf _{u \in \mathcal{L}_{C}} \mathcal{E}_{1}(u, u), & \mathcal{L}_{C} \neq \varnothing \\ \infty, & \mathcal{L}_{C}=\varnothing\end{cases}
$$

denotes the capacity of an open set $C$ (see $[6, \S 2.1]$ ). For an arbitrary set $D$ the capacity is defined in the following way:

$$
C a p(D)=\inf \{C a p(C): D \subset C, C \text { is open }\} .
$$

A positive Borel measure is called smooth, if it charges no set of capacity zero, and there exists a so-called exhausting sequence (see $[6, \S 2.1]$ ). Let $\mu$ be the smooth measure (with respect to $\mathcal{E}(\cdot, \cdot)$ ), with support $F$. Then there exists a positive continuous additive functional $\left(L_{t}\right)_{t \geq 0}$, which is in Revuz correspondence with $\mu$ (see [6]). Let $\check{F}$ be the support of $\left(L_{t}\right)_{t \geq 0}$, and denote by $\sigma_{\breve{F}}$ the first moment when the random process associated with $\left(\mathcal{E}, \mathcal{F}_{e}\right)$ hits $\check{F}$. Since $\mu$ is smooth, and $F-\check{F}$ is $\mu$-negligible, then $\sigma_{\check{F}}<\infty$, see $[5, \S 4.2-4.3]$. Let $H_{\breve{F}} u(x)=E_{x}\left(u\left(X_{\sigma_{\breve{F}}}\right)\right), x \in X$.

Definition 4.1. The time changed Dirichlet form is $(\check{\mathcal{E}}, \check{\mathcal{F}})$, where

$$
\begin{aligned}
\check{\mathcal{F}} & =\left\{\varphi \in L_{2}(F, \mu): \varphi=u \mu \text {-a.e. on } F \text { for some } u \in \mathcal{F}_{e}\right\} \\
\check{\mathcal{E}}(\varphi, \varphi) & =\mathcal{E}\left(H_{\check{F}} u, H_{\check{F}} u\right), \quad \varphi \in \check{\mathcal{F}} .
\end{aligned}
$$

The following Dirichlet principle holds: 
Lemma 4.2. For $\varphi \in \check{\mathcal{F}}, u \in \mathcal{F}_{e}, \check{\mathcal{E}}(\varphi, \varphi)=\inf \{\mathcal{E}(u, u), u=\varphi$ н-a.e. $\}$.

We will show below that starting with a Hunt process associated with $\left(\mathcal{E}, \mathcal{F}_{e}\right)$ we can construct the time-changed process, associated with $(\check{\mathcal{E}}, \check{\mathcal{F}})$, i.e., we can show how this time change is defined. In our situation we even will be able to find $(\check{\mathcal{E}}, \check{\mathcal{F}})$ explicitly.

Now we turn into our situation. Let $A$ be an operator with continuous negative definite symbol $\psi_{0}$, satisfying the sector condition $\left|\operatorname{Im} \psi_{0}\right| \leq c \operatorname{Re} \psi_{0}$. Let $\psi=\operatorname{Re} \psi_{0}$, and consider the symmetrization of the Dirichlet form associated with $-A$, i.e.,

$$
\mathcal{E}_{1}^{\psi}(u, u)=-(\sqrt{\psi} \hat{u}, \sqrt{\psi} \hat{u})+(u, u), \quad \text { with } u \in H^{\psi, 1}\left(=: H_{2}^{\psi, 1}\right) .
$$

Note that in this situation

$$
c\|u\|_{H^{\psi, 1}}^{2} \leq \mathcal{E}_{1}^{\psi}(u, u), \quad u \in H^{\psi, 1}
$$

holds, and therefore $\mathcal{E}_{1}^{\psi}(\cdot, \cdot)$ is equivalent to the scalar product on $H^{\psi, 1}$.

We claim that if the space $H^{\psi, 1}\left(\mathbb{R}^{n}\right)$ admits the trace on $\mathbb{R}^{n-1}$, then $\operatorname{Cap}\left(\mathbb{R}^{n-1}\right)>0$, which is necessary to define a time-changed process on the boundary $\mathbb{R}^{n}$. Indeed, suppose that the space $H^{\psi, 1}\left(\mathbb{R}^{n}\right)$ admits the trace space on $\mathbb{R}^{n-1}$. Let $K$ be a compact subset of $\mathbb{R}^{n-1}$. Then there exists a function $u \in H^{\psi, 1}\left(\mathbb{R}^{n}\right)$ such that $u \geq 1$ on $K \times[0,1]$. Due to the existence of the trace of $u$ on $\mathbb{R}^{n-1}$ we obtain that $u\left(x^{\prime}, 0\right) \geq 1$ on $K$. Then $\mathcal{L}_{K} \neq \varnothing$, and $\operatorname{Cap}(K)<\infty$. By Lemma 3.1.1 from [5] there exists the unique element $e_{K}, 0 \leq e_{K} \leq 1 \mu$-a.e., and $e_{K}=1$ on $K \mu$-a.e., where $\mu$ is the Lebesgue measure on $\mathbb{R}^{n-1}$, and $\mathcal{E}_{1}^{\psi}\left(e_{K}, e_{K}\right)=\operatorname{Cap}(K)$. But then $\operatorname{Cap}(K)>0$, otherwise $e_{K}=0$, since $\mathcal{E}_{1}^{\psi}(\cdot, \cdot)$ is a scalar product on $H^{\psi, 1}\left(\mathbb{R}^{n}\right)$. By monotonicity, $\operatorname{Cap}\left(\mathbb{R}^{n-1}\right) \geq \operatorname{Cap}(K)$.

Thus, $\operatorname{Cap}\left(\mathbb{R}^{n-1}\right)>0$. Then there exists a smooth measure on it. Let $\mu(d x)$ be the Lebesgue measure, $\operatorname{supp} \mu=\mathbb{R}^{n-1}$. It is smooth, therefore there exists a positive continuous additive functional $\left(L_{t}\right)_{t>0}$ which is in Revuz correspondence with $\mu$. In our situation the resolvent measure associated with $\mathcal{E}_{\lambda}^{\psi}$ is absolutely continuous with respect to the Lebesgue measure on $\mathbb{R}^{n}$; denote by $g_{\lambda}(x, y)$ its density. Let $\left(Y_{t}\right)_{t \geq 0}$ be a Hunt process associated with $\mathcal{E}_{1}^{\psi}$. By Theorem 5.1.3 from [6] we get

$$
\int_{\mathbb{R}^{n}} h(x)\left[E_{x}\left(\int_{0}^{\infty} e^{-\lambda t} \phi\left(Y_{t}\right) d L_{t}\right)-\int_{\mathbb{R}^{n}} g_{\lambda}(x, z) \phi(z) \mu(d z)\right] d x=0
$$

for every Borel measurable and positive functions $h$ and $\phi$, which leads to

$$
E_{x}\left[\int_{0}^{\infty} e^{-\lambda t} d L_{t}\right]=\int_{\mathbb{R}^{n}} g_{\lambda}(x, y) \mu(d y)
$$


(note that $\operatorname{supp} \mu=\mathbb{R}^{n-1}$ ). From (4.2) we have for $t(s):=\inf \left\{t: L_{t}(\omega)>s\right\}-$ the right inverse of $L_{t}$ - that

$$
E_{x}\left[e^{-\lambda t(s, \omega)}\right]=e^{-\frac{s}{r_{\lambda}\left(x^{\prime}\right)}}, \quad x^{\prime} \in \mathbb{R}^{n-1},
$$

where $x=\left(x^{\prime}, 0\right), r_{\lambda}\left(x^{\prime}\right)=\int_{\mathbb{R}^{n}} g_{\lambda}(x, y) \mu(d y)$, because $\int_{0}^{\infty} E_{x} e^{-\lambda t(s, \omega)} d u=$ $\int_{0}^{\infty} E_{x} e^{-\lambda s} d L_{s}=r_{\lambda}\left(x^{\prime}\right)$. Since in our case $g_{\lambda}(x, y)$ depends on the difference $x-y$, then $r_{\lambda}\left(x^{\prime}\right)=: r(\lambda)$ does not depend on $x^{\prime} \in \mathbb{R}^{n-1}$. The process $t(s)$ is an increasing compound Poisson process, and therefore it is a subordinator, and the function $\frac{1}{r(\lambda)}$ is its Laplace exponent.

Let $\rho\left(\xi^{\prime}\right)=\frac{1}{r\left(\psi\left(\xi^{\prime}, 0\right)\right)}$. To simplify the notation let $\mathcal{D}=\operatorname{tr}_{\mathbb{R}^{n-1}} H^{\psi, 1}$.

Lemma 4.3. The trace space $\mathcal{D}$ is equivalent to $H^{\rho, 1}\left(\mathbb{R}^{n-1}\right)$.

Proof. Indeed, the Dirichlet form $\mathcal{E}_{1}^{\psi}(u, v)$ is a scalar product on $H^{\psi, 1}$. From Lemma 4.2 and the existence of the retraction and the coretraction on the space $H^{\psi, 1}$ we have for the time changed Dirichlet form

$$
\begin{aligned}
\check{\mathcal{E}}_{1}^{\psi}(v, v) & =\inf \left\{\|u\|_{H^{\psi, 1}}^{2}: u=v \mu \text {-a.e., } u \in H^{\psi, 1}\right\} \\
& \sim \inf \left\{\|u(\cdot, 0)\|_{\mathcal{D}}: u=v \mu \text {-a.e., } u \in H^{\psi, 1}\right\}
\end{aligned}
$$

(where " " means "equivalent"). Therefore $\check{\mathcal{E}}_{1}^{\psi}(v, v)$ is equivalent to the scalar product on $\mathcal{D}$. But $\check{\mathcal{E}}_{1}^{\psi}(v, v)$ corresponds to $\left(\check{Y}_{s}\right)_{s \geq 0}$ - the time changed process with respect to $t(s)$ of $\left(Y_{t}\right)_{t \geq 0}$, i.e., $\check{Y}_{s}=Y_{t(s)}$. And as we saw above the symbol of the generator which corresponds to $\left(\check{Y}_{s}\right)_{s \geq 0}$ is equivalent to $\rho\left(\xi^{\prime}\right), \xi^{\prime} \in \mathbb{R}^{n-1}$. Therefore the Dirichlet form $\check{\mathcal{E}}_{1}^{\psi}(v, v)$ is equivalent to $\mathcal{E}_{1}^{\rho}(v, v)$, which gives us the equivalence of $\mathcal{D}$ and $H^{\rho, 1}$.

Let $\left(X_{t}\right)_{t \geq 0}$ be a Markov process which corresponds to $\left(-A, H_{+}^{\psi, 2}\right)$ with zero Neumann boundary condition. Consider the time changed process $\check{X}_{s}=$ $X_{t(s)}, s \geq 0$. From above, its Laplace exponent is (by subordination) $\rho\left(\xi^{\prime}\right)=$ $\left[r\left(\psi_{0}\left(\xi^{\prime}, 0\right)\right)\right]^{-1}$, and the (non-symmetric) Dirichlet form, associated with $\left(\check{X}_{s}\right)_{s \geq 0}$ is equivalent to $\left(\mathcal{E}^{\rho}, H^{\rho, 1}\right)$, which is a Dirichlet form associated with $\left(\check{Y}_{s}\right)_{s \geq 0}$.

Consider some examples.

Example 4.4. Let $\left(X_{t}\right)_{t \geq 0}$ be a diffusion process with generator $-\triangle$. Then the corresponding Dirichlet form is

$$
\mathcal{E}_{1}(u, v)=\int_{\mathbb{R}^{n}}\left(1+|\xi|^{2}\right) \hat{u}(\xi) \hat{v}(\xi) d \xi,
$$

and its domain is $H_{+}^{1}$. The resolvent kernel associated with $-\triangle$ is

$$
g_{\lambda}(x)=\int_{0}^{\infty} e^{-\lambda t} \frac{e^{-\frac{|x|^{2}}{2 t}}}{(2 \pi t)^{\frac{n}{2}}} d t,
$$


and thus

$$
r(\lambda)=\left.\int_{\mathbb{R}^{n-1}} g_{\lambda}(x) d x^{\prime}\right|_{x_{n}=0}=\left.\int_{0}^{\infty} e^{-\lambda t} \frac{e^{-\frac{\left|x_{n}\right|^{2}}{4 t}}}{(2 \pi t)^{\frac{1}{2}}} d t\right|_{x_{n}=0}=\frac{1}{\sqrt{2 \lambda}} .
$$

Therefore subordinating $\frac{1}{r(\lambda)}=\sqrt{\lambda}$ with $\left|\xi^{\prime}\right|^{2}$ we obtain

$$
\mathcal{E}_{1}^{\rho}(u, v)=\int_{\mathbb{R}^{n-1}}\left(1+\left|\xi^{\prime}\right|\right) \hat{u}\left(\xi^{\prime}\right) \hat{v}\left(\xi^{\prime}\right) d \xi^{\prime}
$$

and thus the trace space of $H^{1}\left(\mathbb{R}^{n}\right)$ (or $\left.H^{1}\left(\mathbb{R}_{+}^{n}\right)\right)$ on $\mathbb{R}^{n-1}$ is $H^{1 / 2}\left(\mathbb{R}^{n-1}\right)$, which is known.

Example 4.5. Consider the subordinate diffusion $\left(X_{t}\right)_{t \geq 0}$ with generator $-(-\triangle)^{\alpha}, \frac{1}{2}<\alpha<1$. Then the corresponding Dirichlet form is

$$
\mathcal{E}_{1}(u, v)=\int_{\mathbb{R}^{n}}\left(1+|\xi|^{2 \alpha}\right) \hat{u}(\xi) \hat{v}(\xi) d \xi
$$

and its domain is $H_{+}^{\alpha}$. The resolvent kernel associated with $-(-\triangle)^{\alpha}$ is

$$
g_{\lambda}(x)=\int_{0}^{\infty} e^{-\lambda t} \int_{0}^{\infty} \frac{e^{-\frac{|x|^{2}}{2 s}}}{(2 \pi s)^{\frac{n}{2}}} \sigma_{\alpha}(s, t) d s d t
$$

where $\left(\sigma_{\alpha}(s, t) d s\right)_{t \geq 0}$ is one-parameter semigroup of measures, which corresponds to the Bernstein function $f(x)=x^{\alpha}, \frac{1}{2}<\alpha<1$. Thus

$$
r(\lambda)=\left.\int_{\mathbb{R}^{n-1}} g_{\lambda}(x) d x^{\prime}\right|_{x_{n}=0}=\left.\int_{0}^{\infty} e^{-\lambda t} \int_{0}^{\infty} \frac{e^{-\frac{\left|x_{n}\right|^{2}}{4 s}}}{(2 \pi s)^{\frac{1}{2}}} \sigma_{\alpha}(s, t) d s d t\right|_{x_{n}=0}=\frac{c_{\alpha}}{\lambda^{1-\frac{1}{2 \alpha}}} .
$$

Therefore subordinating $\frac{1}{r(\lambda)}=\lambda^{1-\frac{1}{2 \alpha}}$ with $\left|\xi^{\prime}\right|^{2 \alpha}$ we obtain

$$
\mathcal{E}_{1}^{\rho}(u, v)=\int_{\mathbb{R}^{n-1}}\left(1+\left|\xi^{\prime}\right|^{2 \alpha-1}\right) \hat{u}\left(\xi^{\prime}\right) \hat{v}\left(\xi^{\prime}\right) d \xi^{\prime}
$$

and thus the trace space of $H^{\alpha}\left(\mathbb{R}^{n}\right)\left(\right.$ or $\left.H^{\alpha}\left(\mathbb{R}_{+}^{n}\right)\right)$ on $\mathbb{R}^{n-1}$ is $H^{\alpha-1 / 2}\left(\mathbb{R}^{n-1}\right)$, which is known.

Example 4.6. Let $f$ be a Bernstein function, such that $f(x) \geq x^{\alpha}, \frac{1}{2}<\alpha<1$, as $x \rightarrow \infty$, and consider $A=-f(-\triangle)$. Then

$$
g_{\lambda}(x)=\int_{0}^{\infty} e^{-\lambda t} \int_{0}^{\infty} \frac{e^{-\frac{|x|^{2}}{2 s}}}{(2 \pi t)^{\frac{n}{2}}} \eta_{t}(d s) d t
$$


where $\eta_{t}(d s)$ is the convolution semigroup which is in one-to-one correspondence with $f$, and thus

$$
\begin{aligned}
r(\lambda) & =\left.\int_{\mathbb{R}^{n-1}} g_{\lambda}(x) d x^{\prime}\right|_{x_{n}=0} \\
& =\left.\int_{0}^{\infty} e^{-\lambda t} \int_{0}^{\infty} \frac{e^{-\frac{\left|x_{n}\right|^{2}}{4 s}}}{(2 \pi s)^{\frac{1}{2}}} \eta_{t}(d s) d t\right|_{x_{n}=0} \\
& =2 \int_{0}^{\infty} \frac{d \zeta}{\left(\lambda+f\left(|\zeta|^{2}\right)\right)} .
\end{aligned}
$$

Thus for $\rho\left(\left|\xi^{\prime}\right|^{2}\right)=\frac{1}{r\left(f\left(\left|\xi^{\prime}\right|^{2}\right)\right)}$ we have

$$
\mathcal{E}_{1}^{\rho}(u, v)=\int_{\mathbb{R}^{n-1}}\left(1+\rho\left(\left|\xi^{\prime}\right|^{2}\right)\right) \hat{u}\left(\xi^{\prime}\right) \hat{v}\left(\xi^{\prime}\right) d \xi^{\prime},
$$

and the trace space of $H_{+}^{f\left(|\cdot|^{2}\right), 1}\left(\mathbb{R}^{n}\right)$ on $\mathbb{R}^{n-1}$ is equivalent to $H^{\rho\left(|\cdot|^{2}\right), 1}\left(\mathbb{R}^{n-1}\right)$.

Remark 4.7. Although the symbols of operators in Examples 4.4-4.6 do not satisfy the assumption $\mathrm{F}$, we still can prove that for such operators the boundary value problem (3.2) is uniquely solvable. In Example 4.4 we have an elliptic operator, and for it the boundary value problem (3.2) is uniquely solvable. In Examples 4.5 and 4.6 the solutions to (3.2) are constructed by subordination, i.e., if

$$
R_{\lambda} g=\int_{0}^{\infty} e^{-\lambda t} T_{t} g d t
$$

is the resolvent for the equation $(\lambda+\triangle) u=g$ (with some boundary condition), where $\left(T_{t}\right)_{t \geq 0}$ is the semigroup generated by $\left(-\triangle, H_{+}^{2}\right)$, then

$$
R_{\lambda}^{f} g=\int_{0}^{\infty} e^{-\lambda t} T_{t}^{f} g d t
$$

is the resolvent for the equation $(\lambda+f(-\triangle)) u=g$ (with some boundary condition), where $f$ is a Bernstein function, such that $f(x) \geq x^{\alpha}, \frac{1}{2}<\alpha<1$, as $x \rightarrow \infty$, and $T_{t}^{f}=\int_{0}^{\infty} T_{s} g(x) \eta_{t}(d s),\left(\eta_{t}(d s)\right)_{t \geq 0}$ is the convolution measure which corresponds to $f$.

Example 4.8. Consider an operator $A$ with symbol $\psi_{0}(\xi)=\left(i \xi_{n}+\left|\xi^{\prime}\right|\right)^{\alpha}$, $\frac{1}{2}<\alpha<1$. Since the domain $H^{\alpha}$ of $A$ admits the trace on $\mathbb{R}^{n-1}$, we see from Theorem 3.2 that $\left(-A, H_{+}^{\psi, 2}\right)$ generates an $L_{2}$-sub-Markovian semigroup. Denote by $\left(X_{t}\right)_{t \geq 0}$ the associated process. But the domain of the corresponding Dirichlet form $H^{\alpha / 2}$ does not admit the trace for $\frac{1}{2}<\alpha<1$. This means that we can construct the process $\left(X_{t}\right)_{t \geq 0}$ with the generator $\left(-A, H_{+}^{\psi, 2}\right)$, but this process with probability 1 will not hit the boundary, it will jump only inside. 
Another probabilistic interpretation of the behaviour of $\left(X_{t}\right)_{t \geq 0}$ is the following. $\left(X_{t}\right)_{t>0}$ is obtained by subordination of the process $\left(Z_{t}\right)_{t>0}$, which corresponds to the symbol $\psi_{1}(\xi)=i \xi_{n}+\left|\xi^{\prime}\right|$, and the subordinator $\left(\tau_{t}\right)_{t \geq 0}$, with the Laplace exponent $f(\lambda)=\lambda^{\alpha}, \frac{1}{2}<\alpha<1$. The term $i \xi_{n}$ in the symbol $\psi_{1}$ stands for a constant-speed movement of the process $\left(Z_{t}\right)_{t \geq 0}$ from the boundary $\mathbb{R}^{n-1}$, while the term $\left|\xi^{\prime}\right|$ stands for the Cauchy process which moves parallel to the boundary. Thus, $\left(Z_{t}\right)_{t>0}$ drifts away from the boundary $\mathbb{R}^{n-1}$ and never hits it; the subordination with $\left(\tau_{t}\right)_{t \geq 0}$ does not change this type of behaviour.

Remark 4.9. Note that the symbols of operators considered in Examples 4.44.8 were obtained by subordination of functions $|\xi|^{2}$ (or $i \xi_{n}+\left|\xi^{\prime}\right|^{2}$ ) with Bernstein functions, and for operators $-A=\triangle$ and $-A=\frac{\partial}{\partial x_{n}}-\triangle_{x^{\prime}}$ the boundary value problem (3.2) is uniquely solvable for Neumann or Dirichlet boundary conditions. Theorem 3.1 says more: it generalizes the class of operators for which we can solve the boundary value problem (3.2) in the half-space.

\section{Some properties of pseudo-differential operators in $L_{p}\left(\mathbb{R}^{n}\right)$}

In this Chapter we will find the conditions under which a pseudo-differential operator $q(x, D)$ with continuous (with respect to $\xi$ ) negative definite symbol $q(x, \xi)$, generates an $L_{p}$-sub-Markovian semigroup in $\mathbb{R}^{n}$.

We start with some auxiliary lemmas, which adjust the results obtained for symbols from Hoh's symbol classes to our situation. For details, see [19], also [11]. For $q \in S_{1,0}^{\psi, m, m^{\prime}}$ we define on $S\left(\mathbb{R}^{n}\right)$ the operator

$$
\begin{aligned}
& q\left(x, D_{x} ; x^{\prime}, D_{x^{\prime}}\right) u(x) \\
& =(2 \pi)^{-\frac{3 n}{2}} \int_{\mathbb{R}^{n}} \int_{\mathbb{R}^{n}} \int_{\mathbb{R}^{n}} e^{i\left(x-x^{\prime}\right) \cdot \xi+i x^{\prime} \xi^{\prime}} q\left(x, \xi ; x^{\prime}, \xi^{\prime}\right) \hat{u}\left(\xi^{\prime}\right) d \xi^{\prime} d x^{\prime} d \xi .
\end{aligned}
$$

Theorem 5.1. Let $\psi$ be an admissible continuous negative definite function, and $q \in S_{1,0}^{\psi, m, m^{\prime}}$. Then for $u \in S\left(\mathbb{R}^{n}\right)$ the iterated integral exists and defines a pseudo-differential operator. The function

$$
q_{L}(x, \xi):=O_{s}-(2 \pi)^{n} \int_{\mathbb{R}^{n}} \int_{\mathbb{R}^{n}} e^{-i y \cdot \eta} q(x, \xi+\eta ; x+y, \xi) d y d \eta
$$

is a symbol in $S_{1,0}^{\psi, m+m^{\prime}}$, and

$$
q\left(x, D_{x} ; x^{\prime}, D_{x^{\prime}}\right) u=q_{L}(x, D) u \quad \text { for } u \in S\left(\mathbb{R}^{n}\right) .
$$

Proof. For $q \in S_{\rho}^{m, \psi}$ the statement was proved in Theorem 2.4.17 from [11], and in such a way (5.2) follows from the embedding $S_{1,0}^{\psi, m} \subset S_{\rho}^{m, \psi}$. We only need to prove that $q_{L}(x, \xi) \in S_{1,0}^{\psi, m+m^{\prime}}$. For more details see also Theorem 2.5, p. 73, from [19]. 
By Peetre's inequality for continuous negative definite functions we have for admissible $\psi$

and since

$$
\frac{(1+\psi(\xi))^{\frac{s}{2}}}{(1+\psi(\eta))^{\frac{s}{2}}} \leq 2^{\frac{|s|}{2}}(1+\psi(\xi-\eta))^{\frac{|s|}{2}}
$$

$$
(1+\psi(\xi)) \leq c\left(1+|\xi|^{2}\right)
$$

holds for any real-valued continuous negative definite function, we get for all $\alpha, \beta \in \mathbb{N}_{0}^{n}$

$$
\begin{aligned}
\left|\partial_{\xi}^{\alpha} \partial_{y}^{\beta} q(x, \xi+\eta ; x+y, \xi)\right| & \leq c_{1} \frac{(1+\psi(\xi+\eta))^{\frac{m}{2}}(1+\psi(\xi))^{\frac{m^{\prime}}{2}}}{\left(1+|\xi+\eta|^{2}\right)^{\frac{|\alpha|}{2}}} \\
& \leq c_{2}\left(1+|\eta|^{2}\right)^{\frac{m}{2}}(1+\psi(\xi))^{\frac{m^{\prime}+m}{2}} .
\end{aligned}
$$

Thus, for fixed $x$ and $\xi$ the function $(\eta, y) \rightarrow q(x, \xi+\eta, x+y, \xi)$ belongs to $\Sigma$, and therefore the oscillatory integral (5.1) exists. Using representation (2.12) we find for sufficiently large $l$ and $l^{\prime}$

$$
\begin{aligned}
\left|\partial_{\xi}^{\alpha} \partial_{x}^{\beta} q_{L}(x, \xi)\right|= & \mid(2 \pi)^{-n} \partial_{\xi}^{\alpha} \partial_{x}^{\beta} \int_{\mathbb{R}^{n}} \int_{\mathbb{R}^{n}} e^{-i y \cdot \eta}\left(1+|y|^{2}\right)^{-l^{\prime}}\left(1-\triangle_{\eta}\right)^{l^{\prime}} \\
& \cdot\left\{\left(1+|\eta|^{2}\right)^{-l}\left(1-\triangle_{y}\right)^{l} q(x, \xi+\eta ; x+y, \xi)\right\} d y d \eta \mid \\
\leq & c_{1} \int_{\mathbb{R}^{n}} \int_{\mathbb{R}^{n}}\left(1+|\eta|^{2}\right)^{-l}\left(1+|y|^{2}\right)^{-l^{\prime}} \frac{(1+\psi(\xi+\eta))^{\frac{m}{2}}}{\left(1+|\xi+\eta|^{2}\right)^{\frac{|\alpha|}{2}}} \\
& \cdot(1+\psi(\xi))^{\frac{m^{\prime}}{2}} d y d \eta \\
\leq & c_{2} \frac{(1+\psi(\xi))^{\frac{m+m^{\prime}}{2}}}{\left(1+|\xi|^{2}\right)^{\frac{|\alpha|}{2}}}
\end{aligned}
$$

i.e., $q_{L}(x, \xi) \in S_{1,0}^{\psi, m+m^{\prime}}$.

Lemma 5.2. Let $\psi$ be an admissible continuous negative definite function, and $q \in S_{1,0}^{\psi, m, m^{\prime}}$. Then $\partial_{\xi}^{\gamma} q\left(x, \xi ; x^{\prime}, \xi^{\prime}\right) \in S_{1,0}^{\psi, m-|\gamma|, m^{\prime}}$.

Proof. Clearly, in view of (5.4) we have

$$
\begin{aligned}
\left|\partial_{\xi}^{\alpha} \partial_{x}^{\beta} \partial_{\xi^{\prime}}^{\alpha^{\prime}} \partial_{x^{\prime}}^{\beta^{\prime}}\left(\partial_{\xi}^{\gamma} q\left(x, \xi ; x^{\prime}, \xi^{\prime}\right)\right)\right| & =\left|\partial_{\xi}^{\alpha+\gamma} \partial_{x}^{\beta} \partial_{\xi^{\prime}}^{\alpha^{\prime}} \partial_{x^{\prime}}^{\beta^{\prime}} q\left(x, \xi ; x^{\prime}, \xi^{\prime}\right)\right| \\
& \leq c_{1} \frac{(1+\psi(\xi))^{\frac{m}{2}}}{\left(1+|\xi|^{2}\right)^{\frac{|\alpha+\gamma|}{2}}} \frac{(1+\psi(\xi))^{\frac{m^{\prime}}{2}}}{\left(1+|\xi|^{2}\right)^{\frac{\left|\alpha^{\prime}\right|}{2}}} \\
& \leq c_{2} \frac{(1+\psi(\xi))^{\frac{m+m^{\prime}-|\gamma|}{2}}}{\left(1+|\xi|^{2}\right)^{\frac{|\alpha|+\left|\alpha^{\prime}\right|}{2}}}
\end{aligned}
$$

which proves the Lemma. 
Lemma 5.3. Let $\psi$ be an admissible continuous negative definite function, and $q \in S_{1,0}^{\psi, m, m^{\prime}}$. Then $q_{L}(x, \xi)-q(x, \xi, x, \xi) \in S_{1,0}^{\psi, m+m^{\prime}-1}$.

Proof. Although the proof is analogous to those of Theorem 3.1, p. 75, from [19], we will give a short outline. By Taylor's formula we have

$$
\begin{aligned}
q(x, \xi+\eta, x+z, \xi)= & q(x, \xi, x+z, \xi) \\
& +\left.\sum_{|\gamma|=1} \eta^{\gamma} \int_{0}^{1} \partial_{\xi}^{\gamma} q\left(x, \xi+t \eta, x+z, \xi^{\prime}\right)\right|_{\xi^{\prime}=\xi} d t
\end{aligned}
$$

where $\eta^{\gamma}:=\eta_{i}$, if $\gamma=(0,0, \ldots, 1,0, \ldots, 0) \in \mathbb{N}_{0}^{n}$. From Theorem 5.1 we derive

$$
\begin{aligned}
(2 \pi)^{-n} q_{L}(x, \xi)= & O_{s}-\int_{\mathbb{R}^{n}} \int_{\mathbb{R}^{n}} e^{-i z \eta} q(x, \xi, x+z, \xi) d z d \eta \\
& +\sum_{|\gamma|=1} O_{s}-\int_{\mathbb{R}^{n}} \int_{\mathbb{R}^{n}} e^{-i z \eta} q_{\gamma}(x, z, \xi, \eta) d \eta \\
= & I_{0}+\sum_{|\gamma|=1} I_{\gamma},
\end{aligned}
$$

where $q_{\gamma}(x, z, \xi, \eta)=\left.\sum_{|\gamma|=1} \eta^{\gamma} \int_{0}^{1} \partial_{\xi}^{\gamma} q\left(x, \xi+t \eta, x+z, \xi^{\prime}\right)\right|_{\xi^{\prime}=\xi} d t$. Clearly, $I_{0}=$ $q(x, \xi ; x, \xi) \in S_{1,0}^{\psi, m+m^{\prime}}$.

Due to (5.3) and (5.4) we find

$$
\begin{aligned}
\left|\partial_{\xi}^{\alpha} \partial_{x}^{\beta} \partial_{\xi^{\prime}}^{\alpha^{\prime}} \partial_{z}^{\beta^{\prime}} q_{\gamma}(x, z, \xi, \eta)\right| & =\left|\int_{0}^{1} \partial_{\xi}^{\alpha} \partial_{x}^{\beta} \partial_{\xi^{\prime}}^{\alpha^{\prime}} \partial_{z}^{\beta^{\prime}} \partial_{\xi}^{\gamma} q\left(x, \xi+t \eta ; x+z, \xi^{\prime}\right)\right|_{\xi^{\prime}=\xi} d t \mid \\
& \leq c_{1} \int_{0}^{1} \frac{(1+\psi(\xi+t \eta))^{\frac{m}{2}}}{\left(1+|\xi+t \eta|^{2}\right)^{\frac{|\alpha|+1-2 l}{2}}} \frac{(1+\psi(\xi))^{\frac{m^{\prime}}{2}}}{\left(1+|\xi|^{2}\right)^{\frac{\left|\alpha^{\prime}\right|}{2}}} d t \\
& \leq c_{2} \frac{(1+\psi(\xi))^{\frac{m+m^{\prime}}{2}}}{\left(1+|\xi|^{2}\right)^{\frac{|\alpha|+1}{2}}}\left(1+|\eta|^{2}\right)^{\frac{|\alpha|+\left|\alpha^{\prime}\right|+1+m}{2}}
\end{aligned}
$$

Then by (2.12) we obtain for $2 l \geq|\alpha|+1+m+n$ and $2 n_{0}>n$

$$
\begin{aligned}
\left|\partial_{\xi}^{\alpha} \partial_{x}^{\beta} I_{\gamma}(x, \xi)\right| & \leq c_{1} \frac{(1+\psi(\xi))^{\frac{m+m^{\prime}}{2}}}{\left(1+|\xi|^{2}\right)^{\frac{|\alpha|+1}{2}}} \int_{\mathbb{R}^{n}} \int_{\mathbb{R}^{n}}\left(1+|\eta|^{2}\right)^{\frac{|\alpha|+1+m}{2}}\left(1+|z|^{2}\right)^{-n_{0}} d z d \eta \\
& \leq c_{2} \frac{(1+\psi(\xi))^{\frac{m+m^{\prime}-1}{2}}}{\left(1+|\xi|^{2}\right)^{\frac{|\alpha|}{2}}}
\end{aligned}
$$

Thus $I_{\gamma} \in S_{1,0}^{\psi, m+m^{\prime}-1}\left(\mathbb{R}^{n}\right)$, and the Lemma is proved. 
Theorem 5.4. Let $\psi$ be an admissible continuous negative definite function, which satisfies $\mathrm{E}$, and $q(x, \xi) \in S_{1,0}^{\psi, 2}$. Then the equation

$$
(\lambda+q(x, D)) u=f,
$$

is uniquely solvable for all $f \in L_{p}, \lambda>0$, and the solution belongs to $H_{p}^{\psi, 2}$.

Proof. First we show that the operator $\left(q_{\lambda}(x, D), H_{p}^{\psi, 2}\right), q_{\lambda}(x, D)=\lambda+q(x, D)$, is closed, i.e., the graph norm of $q_{\lambda}(x, D)$ in $L_{p}$ is equivalent to the norm in $H_{p}^{\psi, 2}$.

Denote by $\left(q_{\lambda}(x, D)\right)^{-1}$ the operator with symbol $\frac{1}{q_{\lambda}(x, \xi)}$, and consider the composition

$$
\begin{aligned}
&\left(q_{\lambda}(x, D)\right)^{-1} \circ q_{\lambda}(x, D) u(x) \\
&:=(2 \pi)^{-\frac{3 n}{2}} \int_{\mathbb{R}^{n}} e^{i x \cdot \xi} q_{\lambda}^{-1}(x, \xi) \int_{\mathbb{R}^{n}} e^{-i x^{\prime} \cdot \xi} \int_{\mathbb{R}^{n}} e^{i x^{\prime} \cdot \xi^{\prime}} q_{\lambda}\left(x^{\prime}, \xi^{\prime}\right) \hat{u}\left(\xi^{\prime}\right) d \xi^{\prime} d x^{\prime} d \xi \\
&=(2 \pi)^{-\frac{3 n}{2}} \int_{\mathbb{R}^{n}} \int_{\mathbb{R}^{n}} \int_{\mathbb{R}^{n}} e^{i\left(x-x^{\prime}\right) \cdot \xi+i x^{\prime} \cdot \xi^{\prime}} \frac{q_{\lambda}\left(x^{\prime}, \xi^{\prime}\right)}{q_{\lambda}(x, \xi)} \hat{u}\left(\xi^{\prime}\right) d \xi^{\prime} d x^{\prime} d \xi \\
&=\tilde{q}_{L}(x, D) u
\end{aligned}
$$

where $\tilde{q}_{L}(x, D)$ is an operator with double symbol $\frac{q_{\lambda}\left(x^{\prime}, \xi^{\prime}\right)}{q_{\lambda}(x, \xi)}$. By Lemma 5.3, $\tilde{q}_{L}(x, \xi)$ can be decomposed as $\tilde{q}_{L}(x, \xi)=1+q^{(1)}(x, \xi)$ with some $q^{(1)} \in S_{1,0}^{\psi,-1}$, (the latter is because $\left.q_{\lambda}^{-1}(x, \xi) \in S_{1,0}^{\psi,-2}\right)$. Due to Theorem 2.9 we obtain for $u \in H_{p}^{\psi, 2}$ that

$$
\begin{aligned}
\|u\|_{H_{p}^{\psi, 2}} & \leq c_{1}\left(\left\|\left(q_{\lambda}(x, D)\right)^{-1} \circ q_{\lambda}(x, D) u\right\|_{H_{p}^{\psi, 2}}+\left\|q^{(1)}(x, D) u\right\|_{H_{p}^{\psi, 2}}\right) \\
& \leq c_{2}\left(\left\|q_{\lambda}(x, D) u\right\|_{L_{p}}+\|u\|_{H_{p}^{\psi, 1}}\right) .
\end{aligned}
$$

Since for all $u \in H_{p}^{\psi, 2}$ the inequality $\|u\|_{H_{p}^{\psi, 1}} \leq \varepsilon\|u\|_{H_{p}^{\psi, 2}}+\frac{c}{\varepsilon}\|u\|_{L_{p}}$ holds for an arbitrary small $\varepsilon>0$ and some positive constant $c$ (see [10, Corollary 4.3.23]), we arrive at

$$
\|u\|_{H_{p}^{\psi, 2}} \leq c_{3}\left(\left\|q_{\lambda}(x, D) u\right\|_{L_{p}}+\|u\|_{L_{p}}\right)
$$

which together with Theorem 2.9 gives

$$
c_{4}\|u\|_{H_{p}^{\psi, 2}} \leq\left\|q_{\lambda}(x, D) u\right\|_{L_{p}}+\|u\|_{L_{p}} \leq c_{5}\|u\|_{H_{p}^{\psi, 2}} .
$$

Therefore $\left(q_{\lambda}(x, D), H_{p}^{\psi, 2}\right)$ is closed.

To prove the solvability of (5.5) we use the Fredholm principle, i.e., the equation $A u=g$, where $(A, D(A))$ is a closed operator in a Banach space $X$, is uniquely solvable for all $g \in X$ if and only if $\operatorname{Ker} A^{*}=\{0\}$, where $A^{*}$ is the adjoint operator. 
Due to Lemma 5.3 we can decompose the symbol of the operator adjoint to $q_{\lambda}(x, D)$ as

$$
q_{\lambda}^{*}(x, \xi)=\overline{q(x, \xi)}+q^{(2)}(x, \xi), \quad \text { where } q^{(2)} \in S_{1,0}^{\psi, 1}
$$

Let $\left(q_{\lambda}^{*}(x, D)\right)^{-1}$ be the operator with symbol $\frac{1}{q_{\lambda}^{*}(x, \xi)} \in S_{1,0}^{\psi,-2}$. By Lemma 5.3

$$
\operatorname{symb}\left(\left(q_{\lambda}^{*}(x, D)\right)^{-1} \circ q_{\lambda}^{*}(x, D)\right)=1+q^{(3)}(x, \xi),
$$

where $q^{(3)} \in S_{1,0}^{\psi,-1}\left(\mathbb{R}^{n}\right)$, and due to Theorem 2.9 for $v \in D\left(q_{\lambda}^{*}(x, D)\right)=L_{p^{\prime}}$ we have

$$
\begin{aligned}
\|v\|_{L_{p^{\prime}}} & \leq \|\left(\left(q_{\lambda}^{*}(x, D)\right)^{-1} \circ q_{\lambda}^{*}(x, D) v\left\|_{L_{p^{\prime}}}+\right\| q^{(3)}(x, D) v \|_{L_{p}^{\prime}}\right. \\
& \leq c_{6}\left(\left\|q_{\lambda}^{*}(x, D) v\right\|_{H_{p^{\prime}}^{\psi,-2}}+\|v\|_{H_{p^{\prime}}^{\psi,-1}}\right) .
\end{aligned}
$$

Suppose that $v$ is such that $q_{\lambda}^{*}(x, D) v=0$. Then from (5.7) we obtain that $v=0$ a.e. in $L_{p^{\prime}}$. Thus $\operatorname{Ker} q_{\lambda}^{*}(x, D)=\{0\}$, and by Fredholm alternative $R\left(q_{\lambda}(x, D)\right)=L_{p}$, which proves the theorem.

Since $\left(-q(x, D), H_{p}^{\psi, 2}\right)$ is an $L_{p}$-Dirichlet operator (by structure), we arrive, using the Hille-Yosida theorem, at

Theorem 5.5. Let $\psi$ be an admissible continuous negative definite function which satisfies $\mathrm{E}, q(x, D)$ be a pseudo-differential operator, the symbol $q(x, \xi)$ of which is a continuous negative definite function with respect to $\xi \in \mathbb{R}^{n}$, and $q \in S_{1,0}^{\psi, 2}$. Then the operator $\left(-q(x, D), H_{p}^{\psi, 2}\right)$ generates an $L_{p}$-sub-Markovian semigroup.

\section{Solvability of the boundary-value problem for some pseudo-differential operator}

In the end we generalize Theorem 3.1 for the case when $A$ is a pseudo-differential operator $q(x, D)$ with continuous negative definite symbol $q(x, \xi)$, satisfying certain restrictions. In what follows we use the notation $q(x, \xi)=q\left(x, \xi^{\prime}, \xi_{n}\right)$, $\xi=\left(\xi^{\prime}, \xi_{n}\right) \in \mathbb{R}^{n}$.

Theorem 6.1. Let $\psi$ be an admissible continuous negative definite function which satisfies $\mathrm{E}, q(x, D)$ be a pseudo-differential operator, the symbol $q(x, \xi)$ of which is a continuous negative definite function with respect to $\xi \in \mathbb{R}^{n}$, and $q \in S_{1,0}^{\psi, 2}$. In addition assume that $q(x, \xi)$ satisfies the condition $\mathrm{F}$ with respect to $\xi_{n}$. If the conditions a) or b) of Theorem 3.1 are satisfied, then $\left(-q(x, D), H_{p,+}^{\psi, 2}\right)$ generates an $L_{p}$-sub-Markovian semigroup. 
Proof. Since the operator $\left(-q(x, D), H_{p}^{\psi, 2}\right)$ satisfies the conditions of Theorem 5.5, it is a generator of an $L_{p}$-sub-Markovian semigroup in $\mathbb{R}^{n}$. The proof of Theorem 6.1 will follow from the proof of Theorem 3.1 if we show that $\lambda+q(x, D)$ is an isomorphism between $H_{p,+}^{\psi, 2}$ and $L_{p}\left(\mathbb{R}_{+}^{n}\right)$.

The conditions of Theorem 3.1 imply that there exists the trace space of $H_{p}^{\psi, 2}$ on $\mathbb{R}^{n-1}$. Then $\tilde{H}_{p,-}^{\psi, 2}$ is the closure of the space $C_{0}^{\infty}\left(\mathbb{R}_{0-}^{n}\right)$ of infinitely many differentiable functions with compact support in $\mathbb{R}_{0 \text { - with respect to the norm }}^{n}$

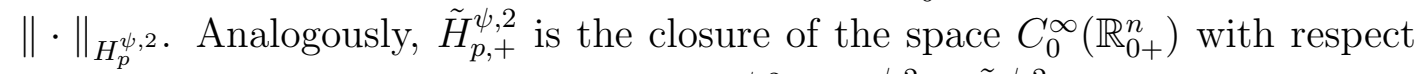
to $\|\cdot\|_{H_{p}^{\psi, 2}}$. Due to the decomposition $H_{p}^{\psi, 2}=H_{p,+}^{\psi, 2} \oplus \tilde{H}_{p,-}^{\psi, 2}$ it is enough to show that for $u \in C_{0}^{\infty}\left(\mathbb{R}_{0-}^{n}\right)$, we have $\operatorname{supp}(\lambda+q(x, D)) u \in \mathbb{R}_{0-}^{n}$, or, to show that for $u \in C_{0}^{\infty}\left(\mathbb{R}_{0+}^{n}\right)$, we have $(\lambda+q(x, D)) u=0$.

We will follow Theorem 2.10.3 from [22], see also [18, 17]. Let $u \in C_{0}^{\infty}(\mathbb{R})$, $\operatorname{supp} u \subset[\varepsilon, \infty)$ for some $\varepsilon>0$, and let $M \in \mathbb{N}$ large enough. Taking the Fourier-Laplace transform of $g$ we derive the estimate

$$
\left|(1+|z|)^{M} \hat{g}(z)\right| \leq C_{g, M} e^{\varepsilon \operatorname{Im} z} .
$$

Consider for $u \in C_{0}^{\infty}\left(\mathbb{R}^{n}\right), \operatorname{supp} u \subset \mathbb{R}^{n} \times[\varepsilon, \infty)$

$$
q(x, D) u(x)=\int_{\mathbb{R}^{n-1}} \int_{-\infty}^{\infty} e^{i \xi^{\prime} \cdot x^{\prime}+i \xi_{n} x_{n}} q\left(x, \xi^{\prime}, \xi_{n}\right) \hat{u}\left(\xi^{\prime}, \xi_{n}\right) d \xi_{n} d \xi^{\prime}
$$

Since the function $\lambda+q\left(x, \xi^{\prime}, z\right)$ has no zeros in the upper half-plane, by Cauchy's theorem we obtain

$$
\begin{aligned}
& (\lambda+q(x, D)) u(x) \\
& \quad=\int_{\mathbb{R}^{n-1}} \int_{i N-\infty}^{i N+\infty} e^{i \xi^{\prime} \cdot x^{\prime}+i x_{n} z}\left(\lambda+q\left(x, \xi^{\prime}, z\right)\right) \hat{u}\left(\xi^{\prime}, z\right) d z d \xi^{\prime} \\
& \quad=\int_{\mathbb{R}^{n-1}} \int_{-\infty}^{+\infty} e^{i \xi^{\prime} \cdot x^{\prime}+i x_{n} \tau-N x_{n}}\left(\lambda+q\left(x, \xi^{\prime}, \tau+i N\right)\right) \hat{u}\left(\xi^{\prime}, \tau+i N\right) d \tau d \xi^{\prime}
\end{aligned}
$$

The condition $q \in S_{1,0}^{\psi, 2}$ implies, taking in account the growth at infinity of a continuous negative definite function, the estimate

$$
|q(x, \xi)| \leq(1+\psi(\xi)) \leq C(1+|\xi|)^{2} .
$$

Then taking $M$ in (6.1) large enough we get

$$
\begin{aligned}
\mid e^{i \xi^{\prime} \cdot x^{\prime}+i x_{n} \tau-N x_{n}}\left(\lambda+q\left(x, \xi^{\prime}, \tau+i N\right)\right) & \hat{u}\left(\xi^{\prime}, \tau+i N\right) \mid \\
& \leq C_{u, M} \frac{C e^{-\left(x_{n}-\varepsilon\right) N}}{\left(1+\left|\xi^{\prime}\right|+\sqrt{\tau^{2}+N^{2}}\right)^{M-2}} .
\end{aligned}
$$


By Lebesgue dominated convergence theorem we obtain for $x_{n}>\varepsilon$

$$
\int_{\mathbb{R}^{n-1}} \int_{-\infty}^{+\infty} e^{i \xi^{\prime} \cdot x^{\prime}+i x_{n} \tau-N x_{n}}\left(\lambda+q\left(x, \xi^{\prime}, \tau+i N\right)\right) \hat{u}\left(\xi^{\prime}, \tau+i N\right) d \tau d \xi^{\prime} \rightarrow 0
$$

as $N \rightarrow \infty$. Thus for $u \in C_{0}^{\infty}\left(\mathbb{R}^{n}\right)$, supp $u \subset \mathbb{R}_{0+}^{n}$, we have $(\lambda+q(x, D)) u=0$, and thus $\lambda+q(x, D)$ is an isomorphism between $\tilde{H}_{p,-}^{\psi, 2}$ and $L_{p}\left(\mathbb{R}_{-}^{n}\right)$, and, consequently, between $H_{p,+}^{\psi, 2}$ and $L_{p}\left(\mathbb{R}_{+}^{n}\right)$.

Acknowledgment. I would like to thank Prof. M. Zähle, Prof. D. Haroske, Prof. H.-G. Leopold, and the participants of seminars on functional spaces, and fractal geometry and stochastics for discussions and remarks. Also I would like to thank the referees for remarks and suggestions. The postdoc DFG grant in Friedrich Schiller University of Jena is gratefully acknowledged.

\section{References}

[1] Blumenthal, R. M. and Getoor, R. K., Markov Processes and Potential Theory. New York: Academic Press 1968.

[2] Farkas, W., Function spaces of generalized smoothness and pseudo-differential operators associated to a continuous negative definite function. Habilitationsschrift, München 2002.

[3] Farkas, W. and Leopold, H.-G., Characterization of function spaces of generalized smoothness. Ann. Mat. Pura Appl. 185 (2006)(1), 1 - 62.

[4] Farkas, W., Jacob, N. and Schilling, R., Function spaces related to continuous negative definite functions: $\Psi$-Bessel potential spaces. Dissertationes Mathematicae CCCXCIII (2001), 1 - 62.

[5] Fukushima, M., Dirichlet Forms and Markov Processes. Amsterdam: NorthHolland 1980.

[6] Fukushima, M., Oshima, Y. and Takeda, M., Dirichlet Forms and Symmetric Markov Processes. Studies in Mathematics 19. Berlin: de Gruyter 1994.

[7] Fukushima, M., Time Changed Markov Processes. Swansea: lecture notes 2003.

[8] Hoh, W., A symbolic calculus for pseudo differential operators generating Feller semigroups. Osaka J. Math. 35 (1998), $798-820$.

[9] Hoh, W., Pseudo differential operators generating Markov processes. Habilitationsschrift, Bielefeld 1998.

[10] Jacob, N., Pseudo-Differential Operators and Markov Processes. Vol. 1: Fourier Analysis and Semigroups. London: Imperial College Press 2001.

[11] Jacob, N., Pseudo-Differential Operators and Markov Processes. Vol.2: Generators and Their Potential Theory. London: Imperial College Press 2002. 
[12] Jacob, N. and Schilling, R., Some Dirichlet spaces obtained by subordinate reflected diffusion. Revista Mat. Iberoamericana 15 (1999)(1), 59 - 91.

[13] Jacob, N. and Schilling, R., Function spaces as Dirichlet spaces - About a paper by Maz'ya and Nagel. Z. Anal. Anwendungen 24 (2005), 3 - 28.

[14] Kaljabin, G. A., Description of traces for anisotropic spaces of TriebelLizorkin type (in Russian). Trudy Mat. Inst. Steklova 150 (1979), 160 - 173.

[15] Kaljabin, G. A., Theorems on extension, multipliers and diffeomorphisms for generalized Sobolev-Liouville classes in domains with Lipschitz boundary (in Russian). Trudy Mat. Inst. Steklov 172 (1985), 173 - 186.

[16] Kaljabin, G. A. and Lizorkin, P. I., Spaces of functions of generalized smoothness. Math. Nachr. 133 (1987), $7-32$.

[17] Knopova, V., Some generators of $L_{p}$-sub-Markovian semigroups in the halfspace $\mathbb{R}_{0+}^{n+1}$. PhD-Thesis, Swansea 2003.

[18] Knopova, V., Semigroups generated by certain pseudo-differential operators on the half-space $\mathbb{R}_{0+}^{n+1}$. Coll. Math. 101 (2004)(2), 221 - 236.

[19] Kumano-go, H., Pseudo-Differential Operators. Cambridge (MA): MIT Press 1974.

[20] Ma, Z.-M. and Röckner, M., An Introduction to the Theory of (Nonsymmetric) Dirichlet Forms. Universitätstext. Berlin: Springer 1992.

[21] Schilling, R. L., Negative definite functions of the form $\varphi(\xi)=f(|\xi|)$. Unpublished manuscript, Erlangen 1997.

[22] Triebel, H., Interpolation Theory, Function Spaces, Differential Operators. North-Holland Mathematical Library 18. Amsterdam: North-Holland 1978.

Received November 29, 2004; revised October 11, 2005 